\title{
Influencia del diseño de la línea de tres puntos sobre el número de jugadoras que participan en posesión del balón y las zonas de lanzamiento en minibasket femenino
}

\author{
Influence of a three-point line shape on the number of players that participate \\ in ball possessions and the shooting zones in girls" mini-basketball
}

\author{
José Luis Arias Estero', Francisco Manuel Argudo Iturriaga ${ }^{1}$, José Ignacio Alonso Roque ${ }^{2}$ \\ 1. Departamento de Educación Física, Deporte y Motricidad Humana. Universidad Autónoma de Madrid \\ 2. Departamento de Ciencias de la Actividad Física y del Deporte. Universidad Católica San Antonio de Murcia
}

CORRESPONDENCIA:

José Luis Arias Estero

Dpto. de Educación Física, Deporte y Motricidad Humana Universidad Autónoma de Madrid

C/ Francisco Tomás y Valiente, 3

28049 Madrid

josel.arias@uam.es

\section{Resumen}

El objetivo de este estudio consistió en comparar dos diseños de la línea de tres puntos en minibasket, para analizar con cuál de los dos aumentaba el número de jugadoras participantes con balón y los lanzamientos a canasta desde el exterior de la zona restringida. Participaron 67 jugadoras, de seis equipos, en dos campeonatos. En el primer campeonato se jugó con la línea de tres puntos demarcada por la zona restringida, y se obtuvieron 1.642 posesiones de balón. En el segundo campeonato se jugó con una línea de tres puntos rectangular similar al área de meta en fútbol, y se obtuvieron 1.669 posesiones de balón. Se utilizó la metodología observacional, mediante un diseño tipo seguimiento, idiográfico, multidimensional. La muestra de los partidos fue total. Tras formar a cuatro observadores, la fiabilidad del registro fue del 0,99. Al jugar con la línea de tres puntos delimitada por el área de la zona restringida se obtuvieron mayores porcentajes, con respecto a la participación en contacto con balón de tres, cuatro y cinco jugadoras $(Z=-3,07, p=0,002)$; y con respecto al número de lanzamientos realizados desde el exterior de la zona restringida y a una distancia superior a cuatro metros $(Z=-3,56, \mathrm{p}=0,000)$.

Palabras clave: Minibasket, línea de tres puntos, modificación de reglas, iniciación deportiva, práctica.

\begin{abstract}
The aim of this study was to compare two shapes of the three-point line in mini-basketball to examine with which of the two shapes a higher number of players took part in ball possessions and a higher number of shots from outside the restricted area were taken. Sixty seven female minibasketball players from six teams participated in two championships. During the first championship, a three-point line delimited by the restricted zone (1,642 ball possessions) was used while during the second one, a rectangular three-point line $(1,669$ ball possessions) was used. Observational methodology was used through a follow-up, ideographic, multidimensional design approach. The sample from matches was total. After training four observers, the register's reliability achieved the value of .99. Greater percentages were observed in playing with the three-point line delimited by the restricted zone with regard to participation in ball possessions by three, four and five players $(Z=-3.07$, $p=.002$ ) and with regard to shots from outside the restricted area that were executed from a distance greater than four meters $(Z=-3.56, p=.000)$.
\end{abstract}

Key words: Mini-basketball, three-point line, rule modification, youth sport, practice. 


\section{Introducción}

En el desarrollo de los niños, la práctica deportiva es esencial porque contribuye al bienestar cognoscitivo, físico, social y emocional (Committee on Sports Medicine and Fitness \& Committee on School Health, 2001; Ginsburg, Committee on Communications \& Committee on Psychosocial Aspects of Child and Family Health, 2007). Sin embargo, para que la práctica deportiva a la que se sometan los niños resulte un estímulo positivo, ésta debe basarse en la reflexión y la comprensión, y en la búsqueda de una mejora integral del individuo a partir de la creación de hábitos motores (Iglesias, Cárdenas \& Alarcón, 2007). Para ello, se debe atender a las necesidades, intereses y posibilidades de los individuos, y no exigir resultados inmediatos (Sáenz-López, Jiménez, Jiménez \& Ibáñez, 2007; Silva, Fernandes \& Celani, 2001). A partir de estas premisas, en la iniciación deportiva resulta necesaria la adquisición de un gran número de experiencias (Côté, Macdonald, Baker \& Abernethy, 2006; MacPhail, Kirk \& Griffin, 2008; Piñar \& Cárdenas, 2004; Silverman, Devillier \& Ramírez, 1991), que sean variadas (Boyce, Coker \& Bunker, 2006; Schmidt, 1975; Williams \& Hodges, 2005), siempre y cuando resulten un estímulo útil para el desarrollo de las habilidades motrices de los niños (Calderón, Palao \& Ortega, 2005; Chalip \& Green, 1998; Dyson, Griffin \& Hastie, 2004; Ntoumanis, Pensgaard, Martin \& Pipe, 2004).

El minibasket es un deporte contextualizado en la iniciación deportiva. Como tal, se debe lograr que el mayor número posible de jugadores participen en contacto con el balón y que adquieran experiencias que les permitan desarrollarse íntegramente (Arias, 2008; Cárdenas \& Pintor, 2001). Una de las habilidades motrices más significativas que el jugador de minibasket debe desarrollar en contacto con el balón es el lanzamiento a canasta. No obstante, para desarrollar esta habilidad de forma óptima, se deben practicar los lanzamientos desde las diferentes zonas de la pista (Maxwell, 2006; Piñar, 2005), incluyendo el exterior de la zona restringida (Arias, Argudo \& Alonso, 2008). Por lo que la participación con balón de la mayor cantidad de jugadores en cada posesión, así como el aumento del número de lanzamientos a canasta desde las diferentes zonas de la pista, denotará una mayor implicación para el desarrollo de las habilidades motrices de los jugadores.

Sin embargo, la evidencia científica ha demostrado que en minibasket la participación con balón se restringe a uno o dos jugadores, que son habitualmente los mismos (Piñar, 2005). Y con respecto a la zona de lanzamiento, se ha comprobado que la mayor parte de los lanzamientos se realizan desde el interior de la zona restringida (Piñar, 2005; Piñar, Alarcón, Vegas, Carreño \& Rodríguez, 2002; Piñar, et al., 2003). Para paliar estos dos aspectos, entre otros, la Federación Española de Baloncesto (FEB) introdujo una línea de tres puntos delimitada por un área rectangular similar al área de meta en fútbol. En este sentido, diferentes federaciones autonómicas han optado por utilizar como línea de tres puntos el área de la zona restringida. Pero no se sabe si al jugar con dos diseños diferentes de la línea de tres puntos las experiencias competitivas son o no diferentes (Arias et al., 2008). Y tampoco se conoce con cuál de las dos opciones se consigue aumentar la participación con balón de los jugadores, así como los lanzamientos desde el exterior de la zona restringida. El objetivo de este estudio consistió en comparar los dos diseños de la línea de tres puntos, para analizar con cuál de los dos aumentaba el número de jugadoras participantes con balón y los lanzamientos desde el exterior de la zona restringida.

\section{Material y Método}

Participantes y muestra

Participaron 67 jugadoras (edad: $M=10,39$ años, SD $=0,67)$, de seis equipos de categoría alevín. Estas jugadoras llevaban practicando minibasket una media de $2,11(\mathrm{SD}=0,77)$ años. Además, las jugadoras practicaban a la semana una media de 5,33 (SD = 1,35) horas, durante una media de $3,5(S D=0,55)$ días. Excepcionalmente, las jugadoras tomaron parte en dos campeonatos organizados con motivo del estudio, en la temporada 2006/2007. La muestra estuvo compuesta por 1.642 posesiones de balón del primer campeonato y 1.669 del segundo. Para seleccionar la muestra de los partidos se utilizó un muestreo total (Anguera, 2003).

Se fijaron dos criterios de inclusión: a) que en los dos campeonatos se debía jugar con las mismas jugadoras y b) que en los 30 días que transcurrieran desde el primer campeonato hasta el segundo, los entrenadores se comprometían a seguir entrenando de forma habitual, pero con la línea de tres puntos rectangular. Antes de proceder a realizar la parte empírica del trabajo, se recogieron los consentimientos informados de los padres y de los responsables de los equipos.

Diseño

Se utilizó la metodología observacional, mediante un diseño tipo seguimiento, idiográfico, multidimensional (Anguera, 2003), para comparar el primer campeonato, que fue cuando se utilizó la línea de tres pun- 


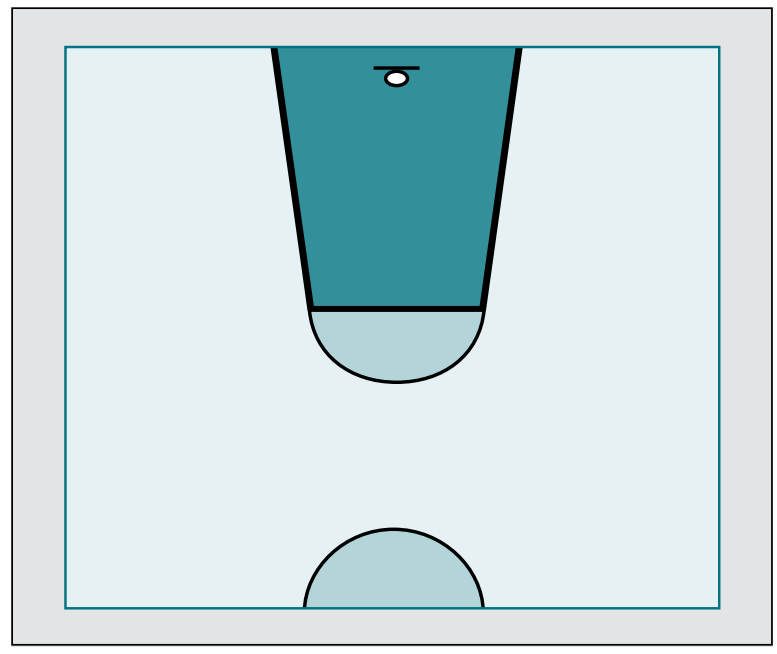

Figura 1. Línea de 3 puntos utilizando la zona restringida.

tos delimitada por el área de la zona restringida (ver Figura 1) y el segundo campeonato, que fue cuando se utilizó la línea de tres puntos delimitada por un área rectangular (ver Figura 2).

En ambos campeonatos la comparación se realizó bajo el análisis de los siguientes criterios:

1. Número de jugadoras que participan en posesión del balón. Se registró el número de jugadoras diferentes que conseguían la posesión del balón cuando su equipo tenía el balón controlado. De manera que en cada posesión de balón, podía haber como mínimo una jugadora participante con balón y como máximo cinco jugadoras.

2. Zona de lanzamiento. Se registró la zona desde la que se ejecutaba cada lanzamiento en cada posesión de balón. Se establecieron las siguientes categorías: (1) zona A, delimitada por el área restringida, (2) zona B, espacio entre el área restringida y la línea de tres puntos rectangular y (3) zona $C$, espacio exterior a la línea de tres puntos rectangular.

\section{Procedimiento}

En primer lugar se procedió a la elaboración del instrumento de observación y del instrumento de registro. Como instrumento de observación se utilizó un sistema de categorías (Anguera \& Blanco, 2003). El proceso de elaboración del sistema de categorías siguió la estrategia empírico-inductiva condicionada por la falta de construcciones teóricas y el carácter multidimensional de la acción (Gorospe, Hernández, Anguera \& Martínez, 2005). Posteriormente, este sistema se perfiló durante la fase de formación de los observadores. El instrumento de registro se construyó a partir de la adaptación de una hoja de cálculo (Microsoft Excel 2003). En el primer campeonato se grabaron nueve partidos

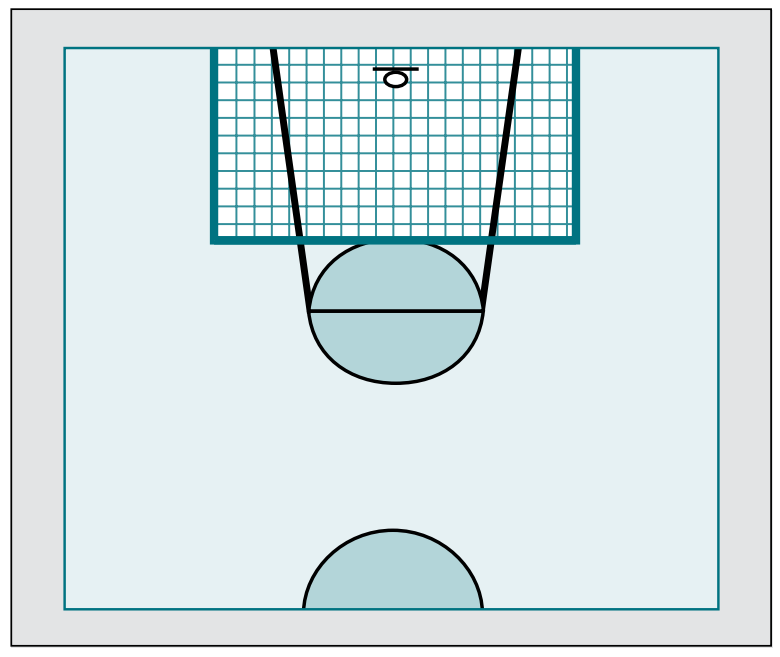

Figura 2. Línea de 3 puntos con forma rectangular.

de competición con dos cámaras de vídeo (JVC, Everio Full HD-GZ-HD7), colocadas transversalmente cada una en un campo de juego. Esto mismo se repitió para el segundo campeonato. Desde el primer campeonato hasta el segundo transcurrieron 30 días. Ambos campeonatos se organizaron con motivo de la investigación y se desarrollaron cada uno a lo largo de un día. En ambos campeonatos se cumplieron los siguientes requisitos de constancia intersesional: a) las jugadoras participantes fueron las mismas, b) se jugaron todos los partidos en el mismo pabellón y dentro de éste en dos pistas idénticas ( $28 \times 15 \mathrm{~m}), \mathrm{c})$ el tiempo de descanso entre partidos fue de diez minutos, d) en cada partido se jugaron seis periodos de ocho minutos cada uno, e) el balón fue del número cinco y f) la altura de las canastas fue de $2,60 \mathrm{~m}$.

Se elaboró un manual que guió el proceso de formación de cuatro observadores. La formación de los observadores siguió las fases de entrenamiento y adiestramiento sugeridas por Anguera (2003). El proceso de formación se produjo en siete sesiones, durante dos semanas. Fruto de este proceso se construyó el manual de instrucciones para los observadores. Una vez finalizado el proceso de formación de los observadores, se obtuvo la fiabilidad del registro a través de la evaluación del rendimiento en relación a un observador experto. El cálculo de la fiabilidad se realizó mediante el coeficiente de concordancia Kappa, alcanzándose el valor de 0,99.

La última parte del procedimiento consistió en la toma de datos mediante un registro sistemático. La observación fue: activa, no participante y directa (Anguera \& Blanco, 2003). En concreto, para aumentar la fiabilidad de la observación, se utilizó la estrategia de observar cada posesión de balón tres veces. La primera vez que se observaba una posesión de balón se hacía 


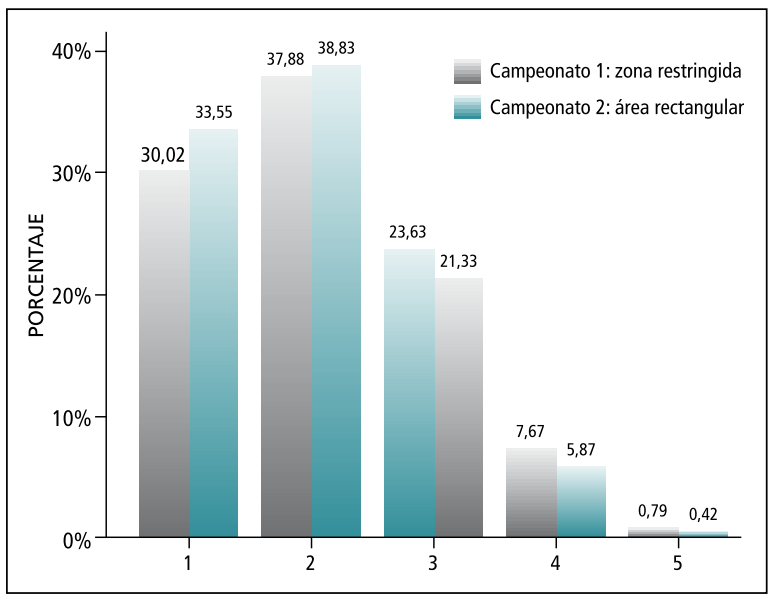

Figura 3. Jugadoras que entraban en contacto con el balón.

a velocidad real del vídeo. Posteriormente, se volvía a observar la misma posesión, dos veces más, a velocidad de 25 frames por segundo para identificar con precisión los criterios a observar. Por último, para cada posesión de balón se registraba la categoría correspondiente a cada criterio.

Los resultados de este estudio están afectados por las ventajas y limitaciones propias de la metodología empleada (Anguera, 2003; Anguera \& Blanco, 2003). Entre las limitaciones destacan los sesgos de observación (Behar \& Riva, 1993). El sesgo de expectancia se solucionó mediante la no comunicación del objetivo del estudio a los observadores. El sesgo mecánico del registro se resolvió mediante la sistematización del proceso y el instrumento de registro. El sesgo de interpretación perceptivo se corrigió mediante la formación de los observadores y la evaluación del rendimiento hasta alcanzar el valor de 0,99 . El sesgo de reactividad se intentó solventar colocando las cámaras de vídeo en la grada (aproximadamente a cinco metros de la pista de juego), para prevenir que las jugadoras se sintieran observadas.

\section{Análisis Estadístico}

Los datos se recogieron mediante el instrumento de registro, para posteriormente capturarlos y archivarlos a través del paquete estadístico SPSS, v. 13.0. para Windows. A través de la prueba U de Mann-Whitney, se analizó si existían diferencias significativas entre las medias de los criterios comparados, atendiendo a las dos situaciones objeto de estudio. Mediante el test de Chi-Cuadrado se conocieron las correlaciones entre los criterios y las dos situaciones, los valores de frecuencia, los porcentajes al jugar con cada línea y los residuos tipificados corregidos. El nivel de significación fue $\mathrm{p}<0,05 \mathrm{y}$ los resultados se obtuvieron con un intervalo de confianza del $95 \%$.

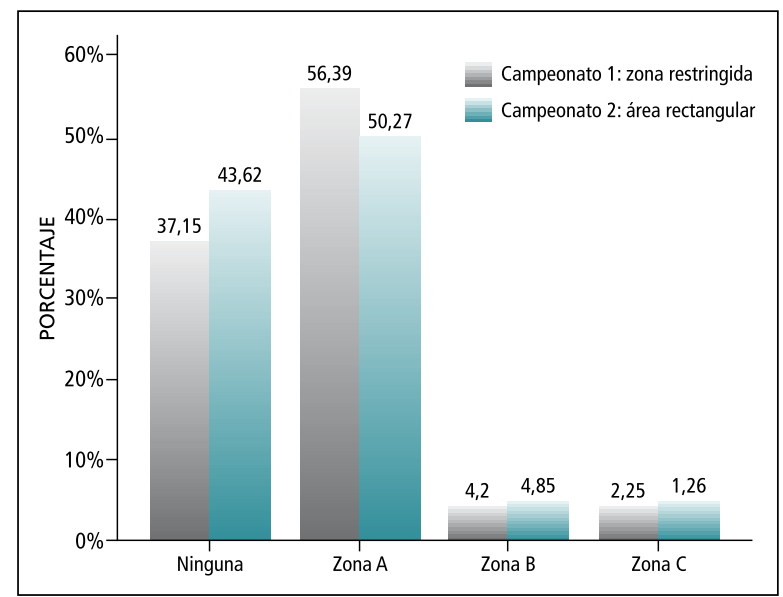

Figura 4. Número de lanzamientos desde cada zona.

\section{Resultados}

Se obtuvieron diferencias significativas entre las dos formas de la línea de tres puntos para el criterio número de jugadoras participantes por posesión de balón $(Z=-3,07, \mathrm{p}=0,002)$, y también para el criterio zona de lanzamiento $(Z=-3,56, \mathrm{p}=0,000)$.

Con respecto al criterio número de jugadoras participantes por posesión de balón, en el primer campeonato participaron en mayor porcentaje tres $(23,6 \%$ frente al $21,3 \%)$, cuatro (7,7\% frente al 5,9\%) y cinco jugadoras $(0,8 \%$ frente al $0,4 \%)$, que en el segundo campeonato $\left(\chi^{2}=11,25\right.$, g.l. $\left.=4, p=0,024\right)$. En el segundo campeonato predominó la participación de una y dos jugadoras (ver Figura 3).

En función del criterio zona de lanzamiento, en el primer campeonato se lanzó a canasta desde el interior de la zona restringida en un $56,4 \%$ de las posesiones de balón. Mientras que en el segundo campeonato esto sucedió en un 50,3\% de las posesiones de balón. Desde el exterior de la zona restringida, se realizaron el 4,2\% de los lanzamientos desde la zona $\mathrm{B}$ y el 2,3\% desde la zona $C$, para el primer campeonato. En el segundo campeonato desde la zona B se ejecutaron el $4,9 \%$ de los lanzamientos y el 1,3\% desde la zona $C$ (ver Figura 4). La relación entre el primer campeonato y la zona de lanzamiento $\mathrm{A}$ y $\mathrm{C}$ resultó directa, mientras que fue inversa para la zona $B\left(\chi^{2}=19,85\right.$, g.l. $\left.=3, p=0,000\right)$.

\section{Discusión}

Según indican Arias et al. (2008), en minibasket no existía evidencia científica de que la inclusión de la línea de tres puntos permitiese el aumento de lanzamientos a canasta desde el exterior de la zona restringida. Igualmente, tampoco se habían encontrado 
trabajos que analizaran las posibles disposiciones y distancias de dicha línea. Por lo que con este estudio se contribuye a esclarecer las limitaciones que existen a la hora de proponer una línea de tres puntos, adaptada a las necesidades formativas y a las características de los jugadores de minibasket. Para favorecer la participación en contacto con el balón y el lanzamiento a canasta desde posiciones exteriores.

Para este estudio hay que considerar que la observación se halla limitada por la duración de los sucesos. Sería interesante que el análisis realizado en este estudio se pudiese prolongar en el tiempo. A pesar de que se realizó una estructuración exhaustiva del escenario experimental, cabe la posibilidad de que interfiriesen factores extraños e incontrolables debido a la naturaleza de la investigación. No obstante, al ser el proceso idéntico en ambos campeonatos, los resultados se ven influenciados por las mismas limitaciones.

En minibasket la participación en contacto con el balón se convierte en uno de los tipos de participación más significativos, puesto que implica la posesión del móvil y por ende que el poseedor sea el responsable directo de mantenerlo, progresar hacia canasta y conseguir encestar. Aunque el objetivo principal del minibasket no sea conservar la posesión del balón, el hecho de obtener el balón permite al jugador la posibilidad de encestar. De manera que los jugadores que más obtengan la posesión del balón serán los que más ejecuten esta habilidad motriz. En este sentido, los valores obtenidos para el criterio número de jugadoras participantes evidenciaron cómo, para ambos campeonatos, en el mayor porcentaje de posesiones participaron en contacto con el balón una y dos jugadoras diferentes. Se observó cómo a medida que aumentaba el número de jugadoras participantes, disminuía el porcentaje de posesiones en las que esto ocurría. Tendencia similar a la observada por Piñar (2005), para minibasket tanto masculino como femenino y para las modalidades $5 \times 5$ y $3 x 3$. Pese a esto, en el primer campeonato el porcentaje de posesiones de balón en las que participaron una o dos jugadoras fue menor, y fue mayor el número de posesiones en las que participaron tres, cuatro y cinco jugadoras con respecto al segundo campeonato y al estudio de Piñar (2005). De manera que al jugar con la línea de tres puntos delimitada por el área de la zona restringida participaron más jugadoras en contacto con el balón por posesión de balón. Lo que supone más posibilidades para ejecutar un número mayor de habilidades en contacto con el balón en dicho campeonato. Y por tanto la acumulación de más horas de práctica significativa en competición, lo que contribuye a desarrollar las habilidades de juego (Baker, Côté \& Abernethy, 2003; Del Villar, Iglesias, Moreno, Fuentes \&
Cervelló, 2004). Con este estudio no se pretende establecer las causas por las que al jugar con la primera línea de tres puntos o con la segunda un número mayor o menor de jugadoras participó en posesión del balón. No obstante, la interacción entre jugadores puede ser interpretada como un proceso emergente resultado de las relaciones espacio-temporales (Passos et al., 2008). Lo que podría explicar el hecho de que al jugar con la línea de tres puntos delimitada por el área de la zona restringida un mayor número de jugadoras obtuvieran la posesión del balón. Este resultado requiere la realización de estudios futuros que analicen el efecto de la modificación del espacio de juego, en general, y de la línea de tres puntos, en particular, sobre la participación de los jugadores. Igualmente, con la finalidad de profundizar en esta relación, sería interesante analizar las estrategias desempeñadas por las jugadoras ante las dos modalidades de la línea de tres puntos.

Cuando el jugador está en posesión del balón y una vez que es capaz de mantenerlo, busca por todos los medios aproximarse a la canasta para lanzar. La aproximación que realice el jugador a la canasta indicará la zona de lanzamiento. La zona de lanzamiento relaciona la distancia y la posición desde donde se produce la ejecución. Al respecto, en el primer campeonato se realizaron un $0,57 \%$ más de lanzamientos desde el interior de la zona restringida en relación con el segundo campeonato. Desde la zona B, en el segundo campeonato se realizaron un $0,7 \%$ más de lanzamientos que en el primero. $\mathrm{Y}$ desde el exterior de la línea de tres puntos delimitada por la FEB, se ejecutaron un $1 \%$ más de lanzamientos en el primer campeonato con respecto al segundo. En cuanto a los lanzamientos ejecutados desde el interior de la zona restringida, los resultados obtenidos en este estudio son superiores a los que indica la bibliografía. En este sentido, Piñar et al. (2002) en minibasket masculino, observan unos valores del $81 \%$. Piñar et al. (2003) obtienen, también en minibasket masculino, un 69,5\% de lanzamientos. Mientras que Piñar (2005), encuentra para minibasket femenino un 71,6\% de lanzamientos para la modalidad 5x5. Por lo que, de acuerdo con estos autores, en las etapas de iniciación, los lanzamientos realizados desde fuera de la zona restringida son escasos, y los lanzamientos lejanos, inexistentes. A pesar de dicha evidencia, en el primer campeonato se lanzó más desde el exterior de la zona restringida y desde una distancia superior a cuatro metros. Lo que contribuye al planteamiento de un proceso de enseñanza que debe ser acorde a las necesidades e intereses propios de la etapa (Chalip \& Green, 1998; Dyson, et al., 2004; Graça \& Oliveira, 1997; Lapresa, Amatria, Egüén, Arana \& Garzón, 2008), al aumentar la variabilidad de las zonas de lanzamiento. 


\section{Conclusión}

El objetivo de este estudio consistió en comparar los dos diseños de la línea de tres puntos, para analizar con cuál de los dos aumentaba el número de jugadoras participantes con balón y los lanzamientos desde el exterior de la zona restringida. Los resultados muestran que al jugar con la línea de tres puntos delimitada por el área de la zona restringida aumenta el número de participaciones de tres, cuatro y cinco jugadoras con respecto a cuando se juega con la línea de tres puntos delimitada por la zona rectangular. Además, en el primer campeonato se obtiene una mayor variabilidad en cuanto a las zonas de lanzamiento y en cuanto a un porcentaje superior de lanzamientos realizados desde el exterior de la zona restringida, en comparación con el segundo campeonato. Por lo que, de acuerdo con la bibliografía, al jugar con la línea de tres puntos delimitada por el área de la zona restringida, se genera un contexto de práctica que permite en mayor medida el desarrollo de las experiencias de juego de las jugadoras y del lanzamiento a canasta. No obstante, es necesario realizar más estudios, tanto con niños como con niñas, para confirmar estos datos, así como para establecer una línea de tres puntos en minibasket $u$ otras adaptaciones que favorezcan el desarrollo de las habilidades de los jugadores.

\section{B I B L IO G R A Fí A}

Anguera, M.T. (2003). La observación. En: C. Moreno Rosset (Ed.), Evaluación psicológica. Concepto, proceso y aplicación en las áreas del desarrollo y de la inteligencia (pp. 271-308). Madrid: Sanz y Torres.

Anguera, M.T. \& Blanco, A. (2003). Registro y codificación en el comportamiento deportivo. En A. Hernández Mendo (Coord.), Psicología del Deporte (Vol. 2). Metodología (pp. 6-34). Buenos Aires: Efdeportes [Reimpreso en A. Hernández Mendo (Coord.) (2005). Psicología del Deporte, Vol. II, Metodología (pp. 33-66). Sevilla: Wanceulen].

Arias, J.L. (2008). El proceso de formación deportiva en la iniciación a los deportes colectivos fundamentado en las características del deportista experto. Retos. Nuevas Tendencias en Educación Física, Deporte y Recreación, 13, 28-32.

Arias, J.L., Argudo, F.M. \& Alonso, J.I. (2008). La inclusión de la línea de tres puntos en minibasket. Revista Internacional de Ciencias del Deporte, 4(13), 54-68.

Baker, J., Côté, J. \& Abernethy, B. (2003). Sport-specific practice and the development of expert decision-making in team ball sport. Journal of Applied Sport Psychology, 15, 12-25.

Behar, J. \& Riba, C. (1993). Sesgos del observador y de la observación. En M.T. Anguera (Ed.), Metodología observacional en la investigación psicológica Vol. II (pp. 11-148). Barcelona: PPU.

Boyce, B.A., Coker, C.A. \& Bunker, L.K. (2006). Implications for variability of practice from pedagogy and motor learning perspectives: finding a common ground. Quest, 58, 330-343.

Calderón, A., Palao, J.M. \& Ortega, E. (2005). Incidencia de la forma de organización sobre la participación, el feedback impartido, la calidad de las ejecuciones y la motivación en la enseñanza de habilidades atléticas. Cultura, Ciencia y Deporte, 1(3), 145-155.

Cárdenas, D. \& Pintor, D. (2001). La iniciación al baloncesto en el medio escolar. En F. Ruiz, A. García \& A.J. Casimiro (Eds.), La iniciación de portiva basada en los deportes colectivos. Nuevas tendencias metodológicas (pp. 105-143). Madrid: Gymnos.

Chalip, L. \& Green, B.C. (1998). Establishing and maintaining a modified youth sport program: lessons from hotelling's location game. Sociology of Sport Journal, 15, 326-342.

Committee on Sports Medicine and Fitness \& Committee on School Health (2001). Organized sports for children and preadolescents. Pediatrics, 107(6), 1459-1462.

Côté, J., Macdonald, D.J., Baker, J. \& Abernethy, B. (2006). When "where" is more important than "when": birthplace and birthdate effects on the achievement of sporting expertise. Journal of Sports Sciences, 24(10), 1065-1073.

Del Villar, F., Iglesias, D., Moreno, M.P., Fuentes, J.P. \& Cervelló, E.M (2004). An investigation into procedural knowledge and decisionmaking: spanish experienced-inexperienced basketball players differences. Journal of Human Movement Studies, 46, 407-420.

Dyson, B., Griffin, L.L. \& Hastie, P. (2004). Sport education, tactical games, and cooperative learning: theoretical and pedagogical considerations. Quest, 56, 226-240.

Ginsburg, K.R., Committee on Communications \& Committee on Psychosocial Aspects of Child and Family Health. (2007). The importance of play in promoting healthy child development and maintaining strong parent-child bonds. Pediatrics, 119(1), 182-191.
Gorospe, G., Hernández, A., Anguera, M.T. \& Martínez, R. (2005). Desarrollo y optimización de una herramienta observacional en el tenis de individuales. Psicothema, 17(1), 123-127.

Graça, A. \& Oliveira, J. (1997). La enseñanza de los juegos deportivos. Barcelona: Paidotribo.

Iglesias, D., Cárdenas, D. \& Alarcón, F. (2007). La comunicación durante la intervención didáctica del entrenador. Consideraciones para el desarrollo del conocimiento táctico y la mejora en la toma de decisiones en baloncesto. Cultura, Ciencia y Deporte, 3(7), 43-50.

Lapresa, D., Amatria, M., Egüén, R., Arana, J. \& Garzón, B. (2008). Análisis descriptivo y secuencial de la fase ofensiva del fútbol 5 en la categoría prebenjamín, Cultura, Ciencia y Deporte, 8(3), 107-116.

MacPhail, A., Kirk, D. \& Griffin, L. (2008). Throwing and catching as relational skills in game play: Situated learning in a modified game unit. Journal of Teaching in Physical Education, 27, 100-115.

Maxwell, T. (2006). A progressive decision options approach to coaching invasion games: basketball as an example. Journal of Physical Education New Zealand, 39(1), 58-71.

Ntoumanis, N., Pensgaard, A.-M., Martin, C. \& Pipe, K. (2004). An idiographic analysis of amotivation in compulsory school physical education. Journal of Sport and Exercise Psychology, 26, 197-214.

Piñar, M.I. (2005). Incidencia del cambio de un conjunto de reglas de juego sobre algunas de las variables que determinan el proceso de formación de lo jugadores de minibasket (9-11 años). Granada: Universidad de Granada.

Piñar, M.I. \& Cárdenas, D. (2004). El minibasket 3x3 y sus aportaciones al juego del niño durante la competición. En A. López, C. Jiménez \& C. López (Eds.), II Curso de Didáctica del baloncesto en las etapas de formación (pp. 16-37). Madrid: Editores.

Piñar, M.I., Alarcón, F., Palao, J.M., Vegas, A., Miranda, M.T. \& Cárdenas, D. (2003). Análisis del lanzamiento en el baloncesto de iniciación. En A. Oña \& A. Bilbao (Eds.), Libro de Actas del II Congreso Mundial de Ciencias de la Actividad Física y el Deporte. Deporte y Calidad de Vida (pp. 202-208). Granada: Editores.

Piñar, M.I., Alarcón, F., Vegas, A., Carreño, F. \& Rodríguez, D. (2002). Posiciones y distancias de lanzamiento durante la competición en minibasket. En A. Díaz, P.L. Rodríguez \& J.A. Moreno (Coords.), Actas del III Congreso Internacional de Educación Física e Interculturalidad. Murcia: Consejería de Educación y Cultura de la Región de Murcia.

Sáenz-López, P., Jiménez, A.C., Giménez, F.J. \& Ibáñez, S.J. (2007). La autopercepción de las jugadoras de baloncesto expertas respecto a sus procesos de formación. Cultura, Ciencia y Deporte, 3(7), 35-41.

Schmidt, R.A. (1975). A schema theory of discrete motor skill learning. Psychological Review, 82, 225-260.

Silva, F.M., Fernandes, L. \& Celani, F.O. (2001). Desporto de crianças e jovens - um estudo sobre as idades de iniciação. Revista Portuguesa de Ciências do Desporto, 1(2), 45-55.

Silverman, S., Devillier, R. \& Ramírez, T. (1991). The validity of academy learning time-physical education (ALT-PE) as a process measure of achievement. Research Quarterly for Exercise and Sport, 62(3), 319-325.

Williams, A.M. \& Hodges, N.J. (2005). Practice, instruction and skill acquisition in soccer: challenging tradition. Journal of Sports Sciences, 23(6), 637-650 